\title{
USER INTERFACE DEVELOPMENT
}

$\begin{array}{ccc}\begin{array}{c}\text { Bharat Aggrawal } \\ \begin{array}{c}\text { Mechanical Technology, Inc. } \\ \text { Latham, New York }\end{array}\end{array} & 07174 & 23070 \\ & 322143 & \end{array}$

\section{Curront status}

User interface complete for the OS/2 version of the following components:

- Executive Shell

- Spiral Groove Gas Cylindrical Seals (SPIRALGC)

- Spiral Groove Gas Face Seals (SPIRALGF)

- Spiral Groove Face Seal Optimization (FACE)

- Gas Cylindrical Seals (GCYL)

- Gas Face Seals (GFACE)

- Incompressible Cylindrical Seals (ICYL)

- Incompressible Face Seals (IFACE)

- Fluid Properties Calculation (FLUID)

- Plotting Program for GCYL, GFACE, ICYL, and IFACE.

- Cylindrical Seals Configurations for SCISEAL

\section{New Fealures}

Features added since the last workshop based on user feedback:

- Units conversion between SI and English units from the Analysis Options menu item

- A Set Defaults menu option to set all input values to program defaults

- A Batch Mode option in the Analysis menu to run multiple test cases

- Automatic handling of data files from a previous test case

- Deletion of Input and Output files from the File menu in the Executive

- All analysis codes built using a 32-bit FORTRAN compiler for OS/2. Codes run at least twice as fast as the previous versions. Users must have $O S / 22.0$ or later versions.

- New, easy to use installation program

- Several internal enhancements to improve performance and reduce development time 


\section{System Executive}

i) CFo nothlal codes Exedu

File Setup Holp

\section{CFD Seal Categories}

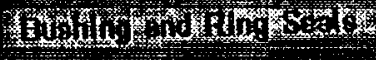

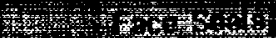
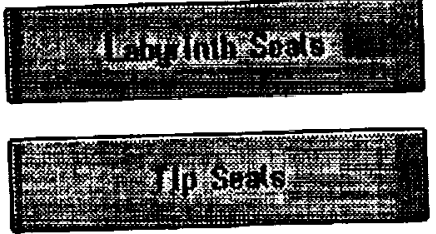

t

Clck On A Seal Calegory
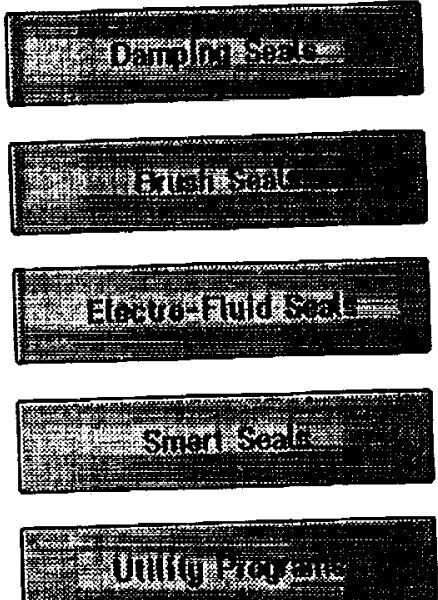

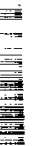

\section{Bushing and Ring Seal Codes}

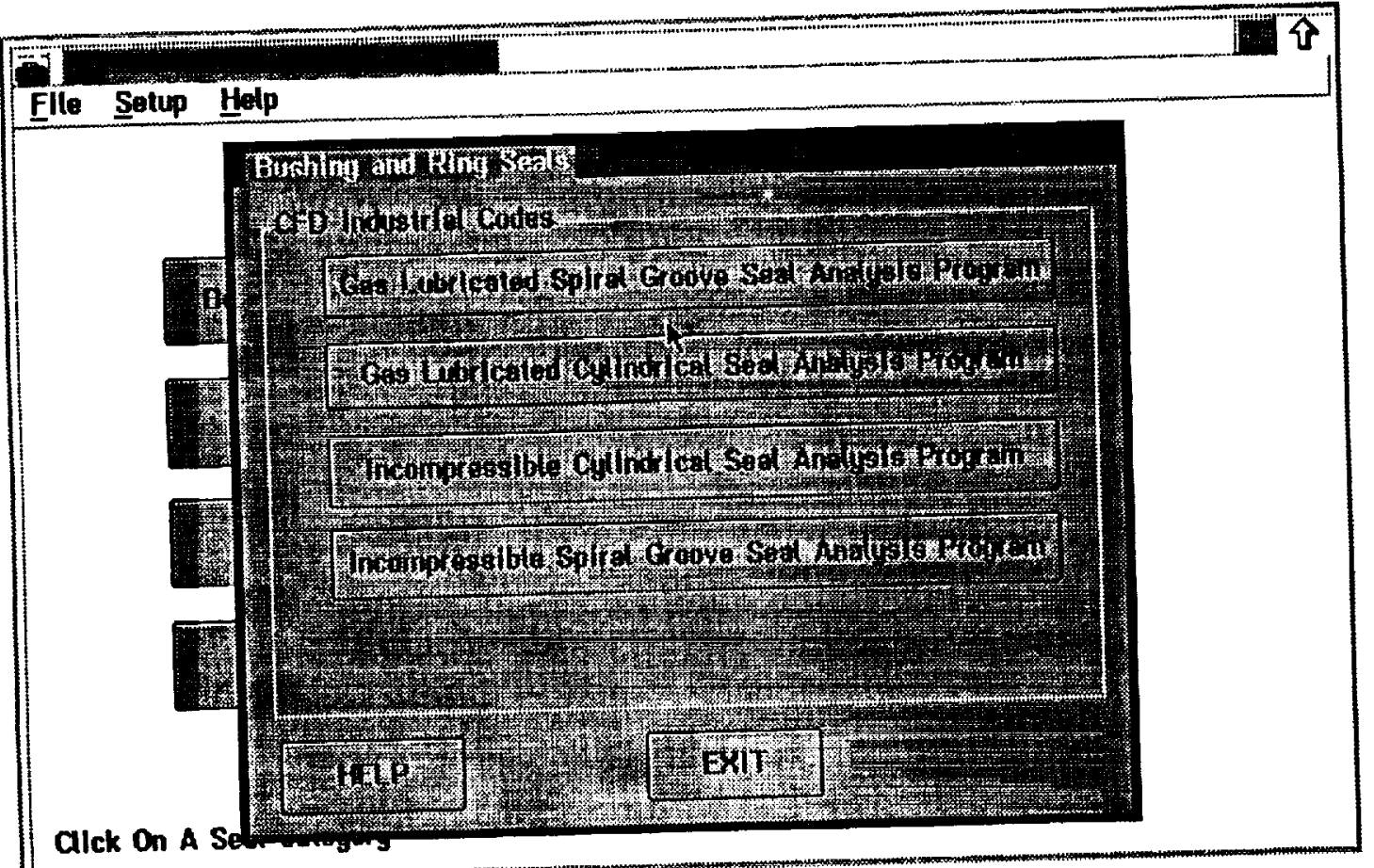


Usingan Industrial Code: FILE menu

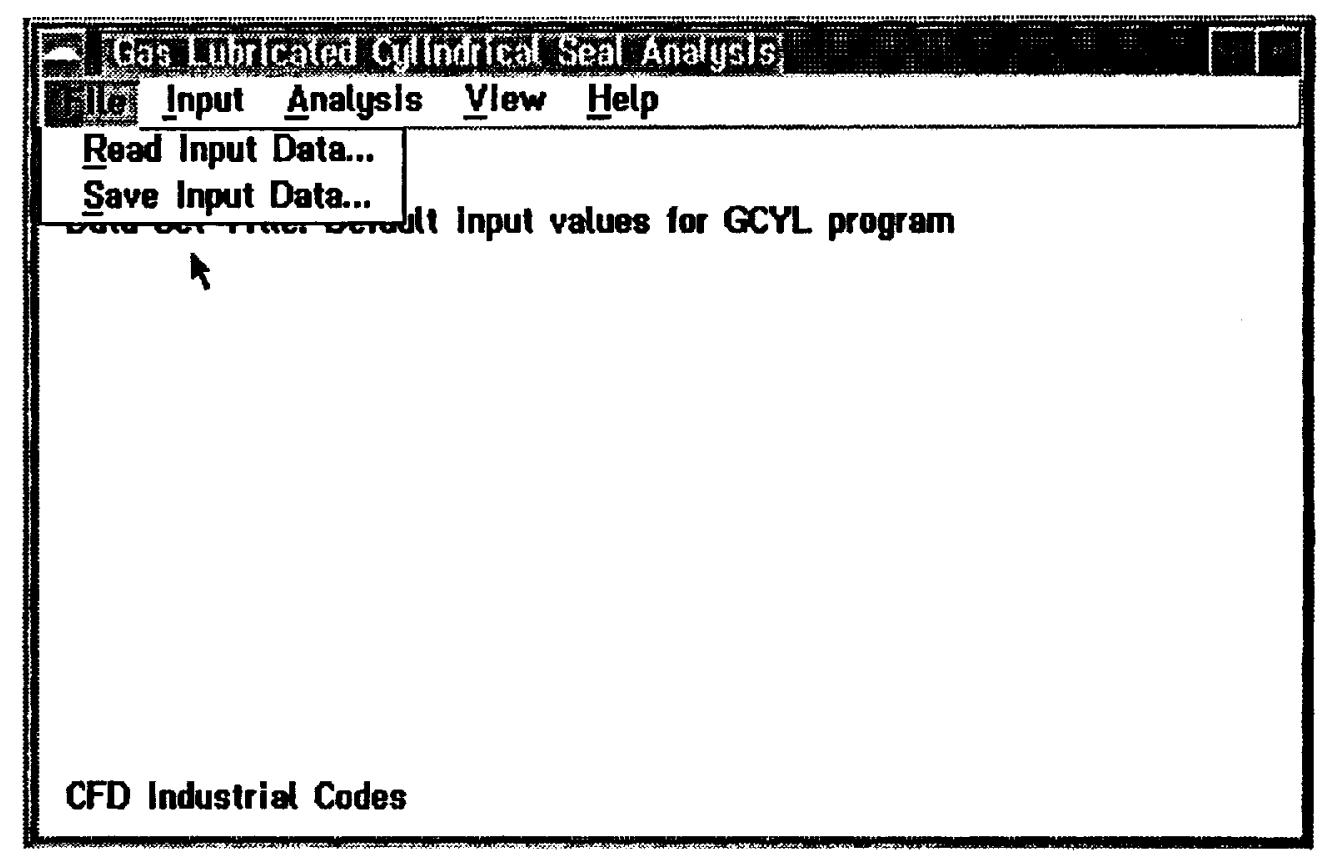

Using an Industrial Code: INPUT menu

\begin{tabular}{|c|c|c|}
\hline \multicolumn{3}{|c|}{ 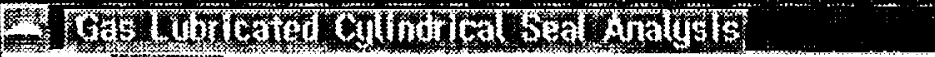 } \\
\hline Flle & Wintit Analysis Vlew & Jelp \\
\hline \multirow[t]{3}{*}{ Data } & $\begin{array}{l}\text { Analysis Options... } \\
\text { Seal Geometry... } \\
\text { Operating Conditions... }\end{array}$ & fes for GCYL program \\
\hline & $\begin{array}{l}\text { S. } \\
\text { Grid Definition... } \\
\text { Properties... } \\
\text { Solution Control... }\end{array}$ & \multirow{2}{*}{$\begin{array}{l}\text { Flow Lines... } \\
\text { Constant Pressure... } \\
\text { Fluid Sources... } \\
\text { Rayleigh Steps... } \\
\text { Recesses... } \\
\text { Spot Recesses... }\end{array}$} \\
\hline & Set Defaults... & \\
\hline \multicolumn{3}{|c|}{ CFD Industrial Codes } \\
\hline
\end{tabular}



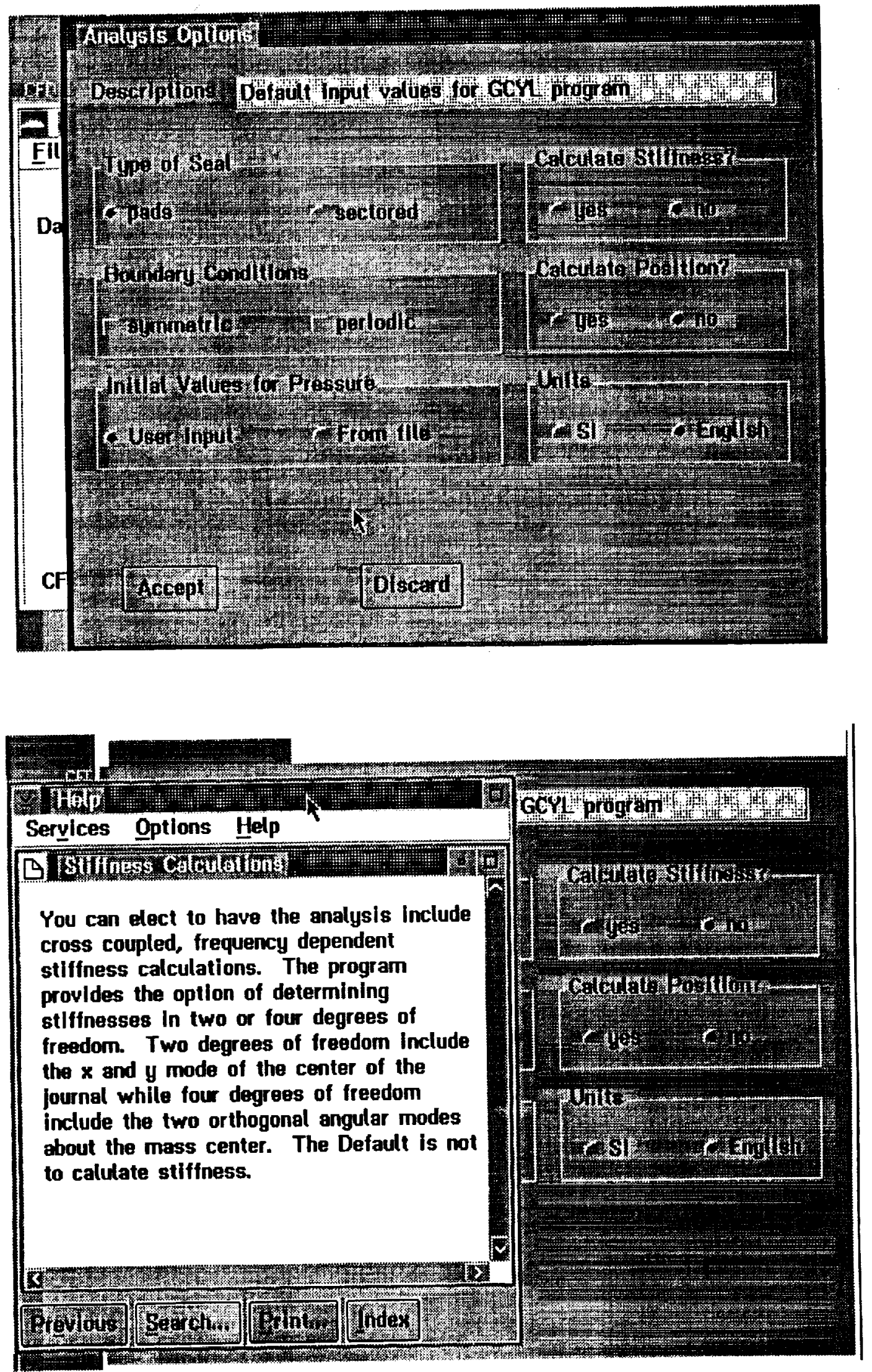


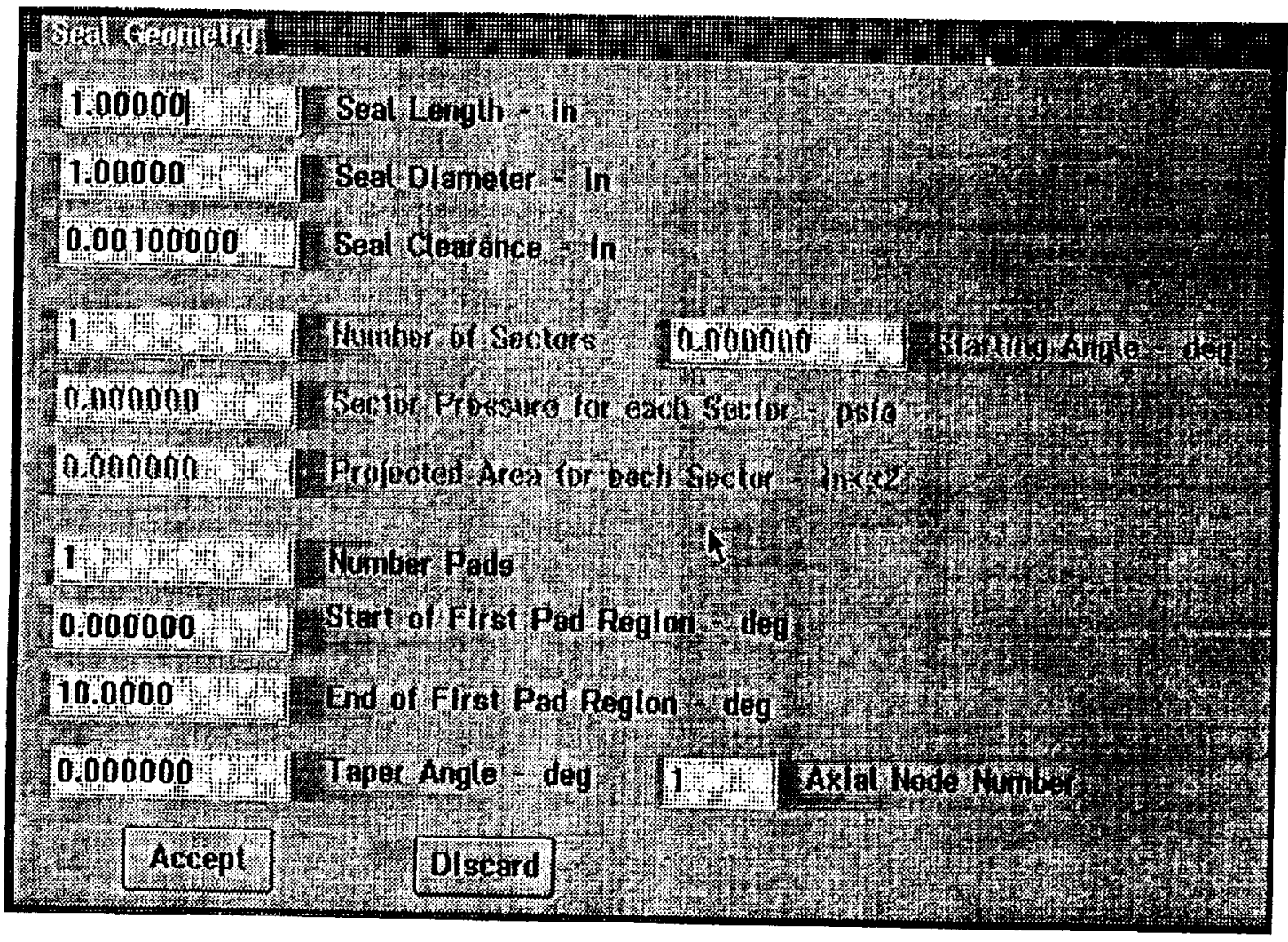

Using an Industrial Codo: Array Input

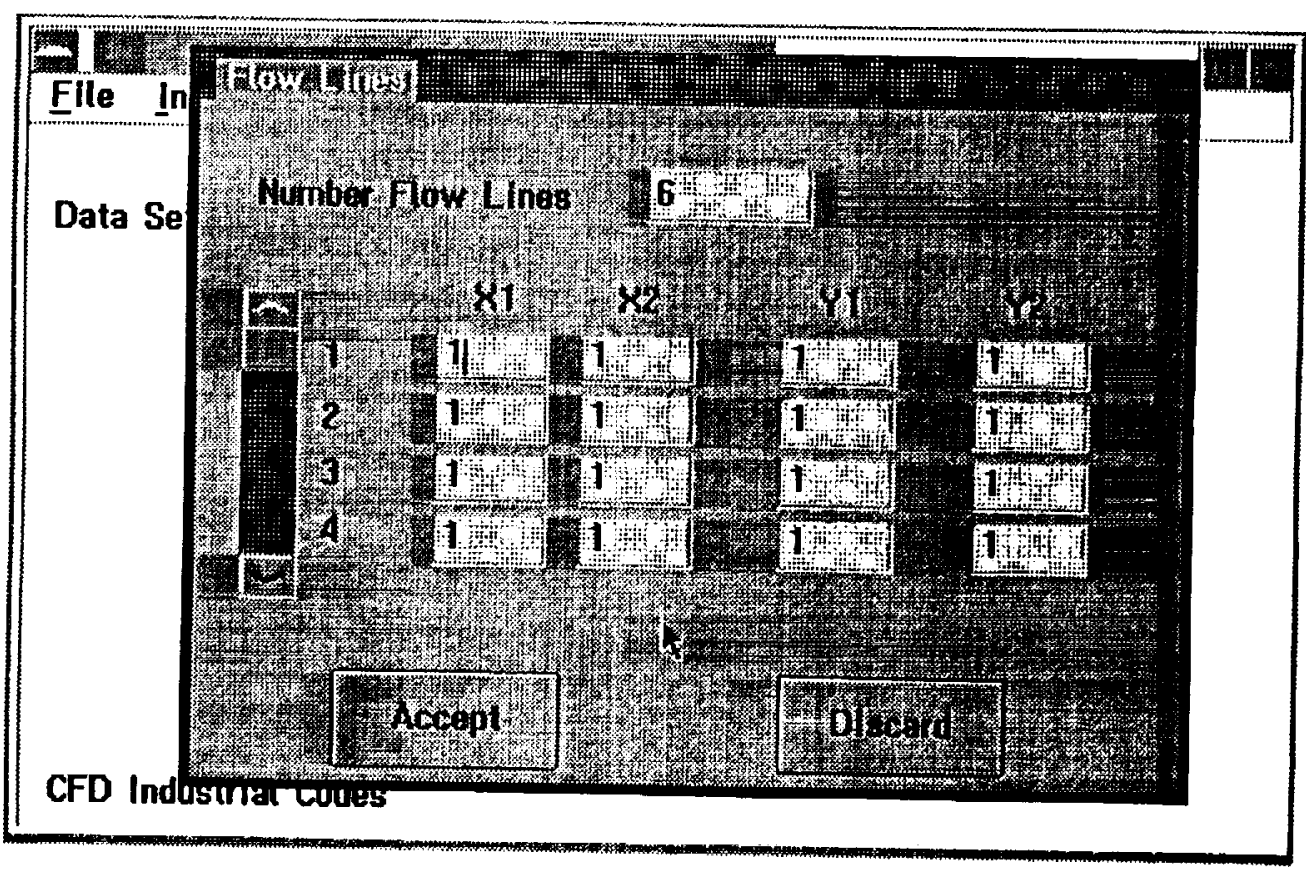


Using an Industrial Code: Analysis menu

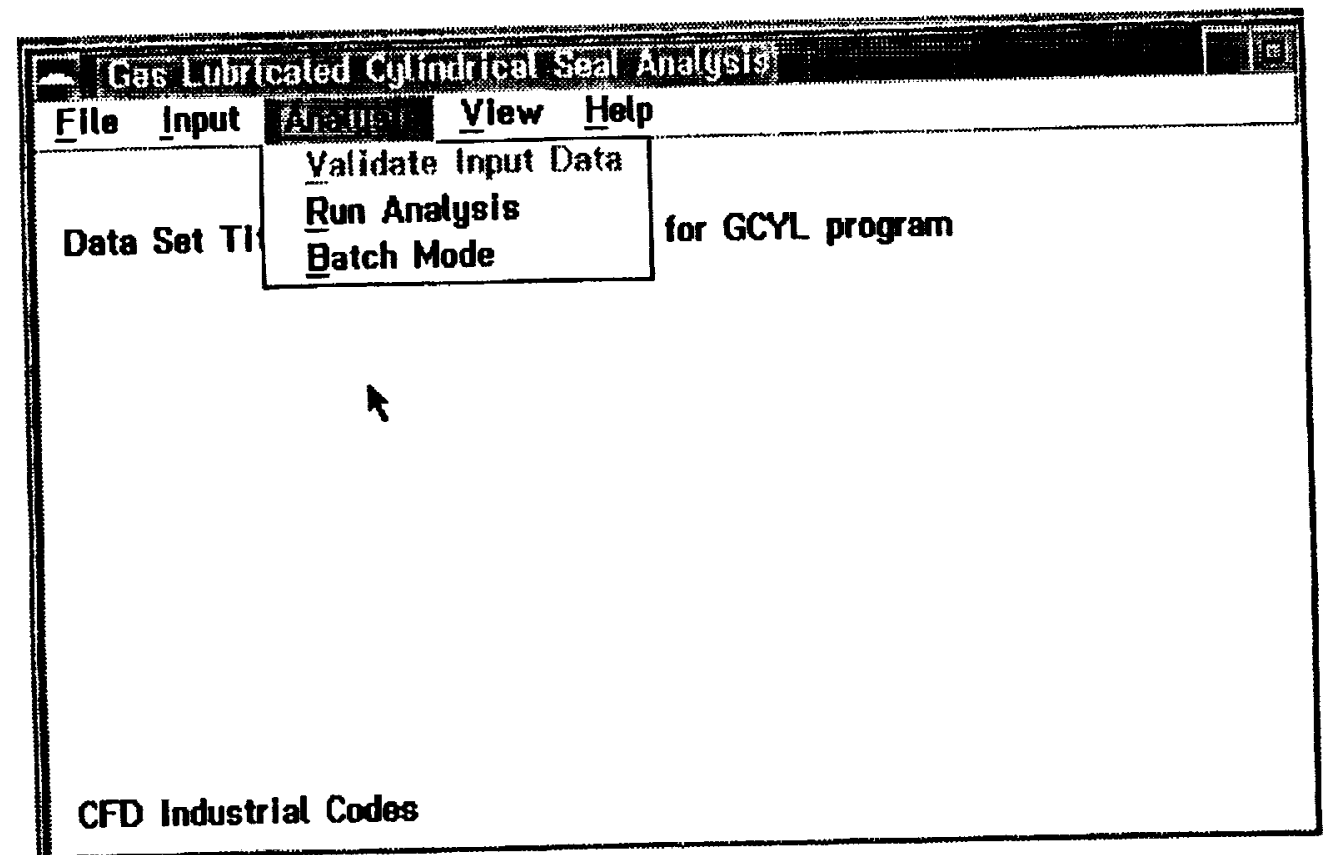

Using an Industrlal Code: Batch Mode

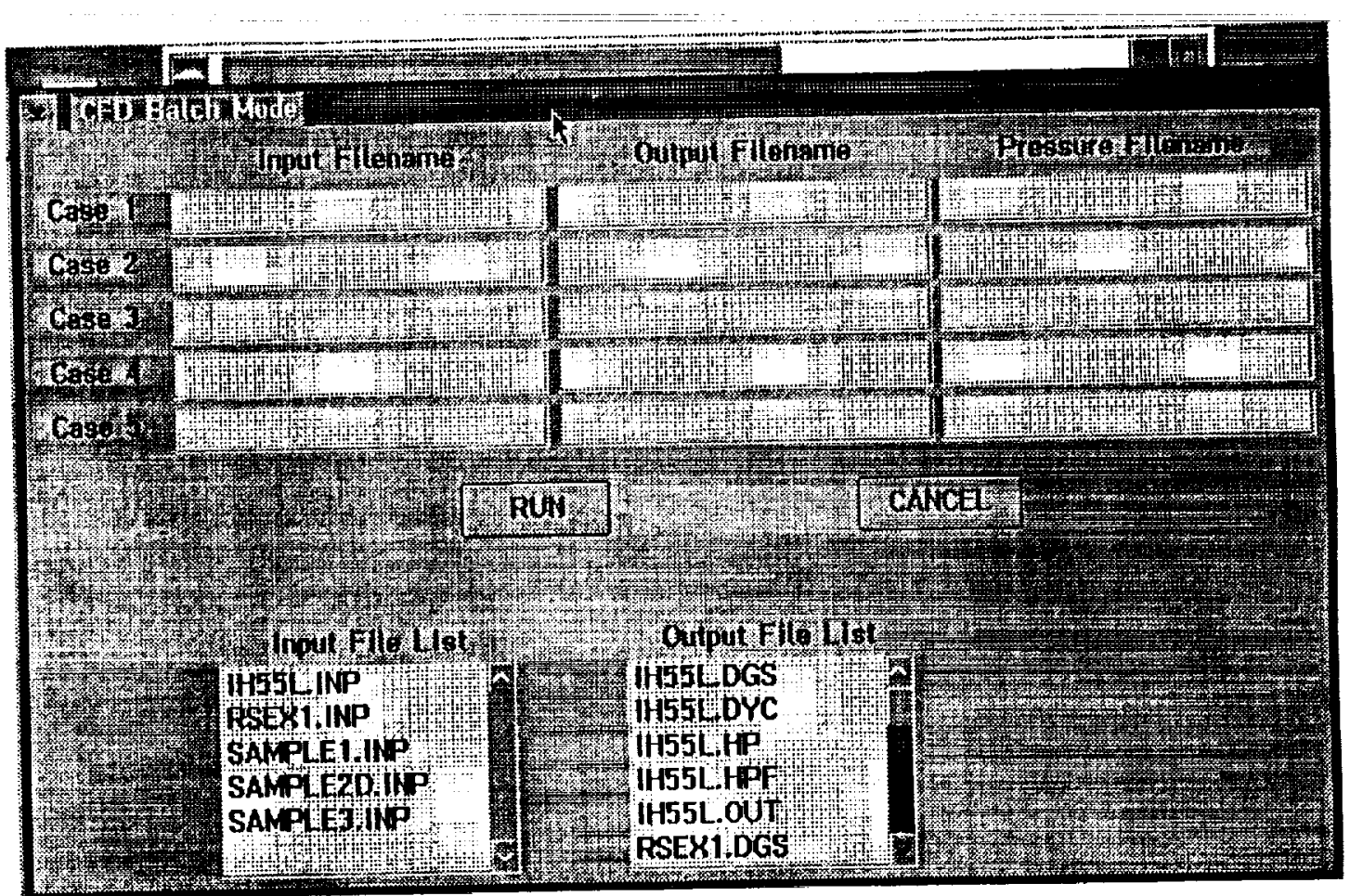


Usingan Industrial Codo: VIEW monu



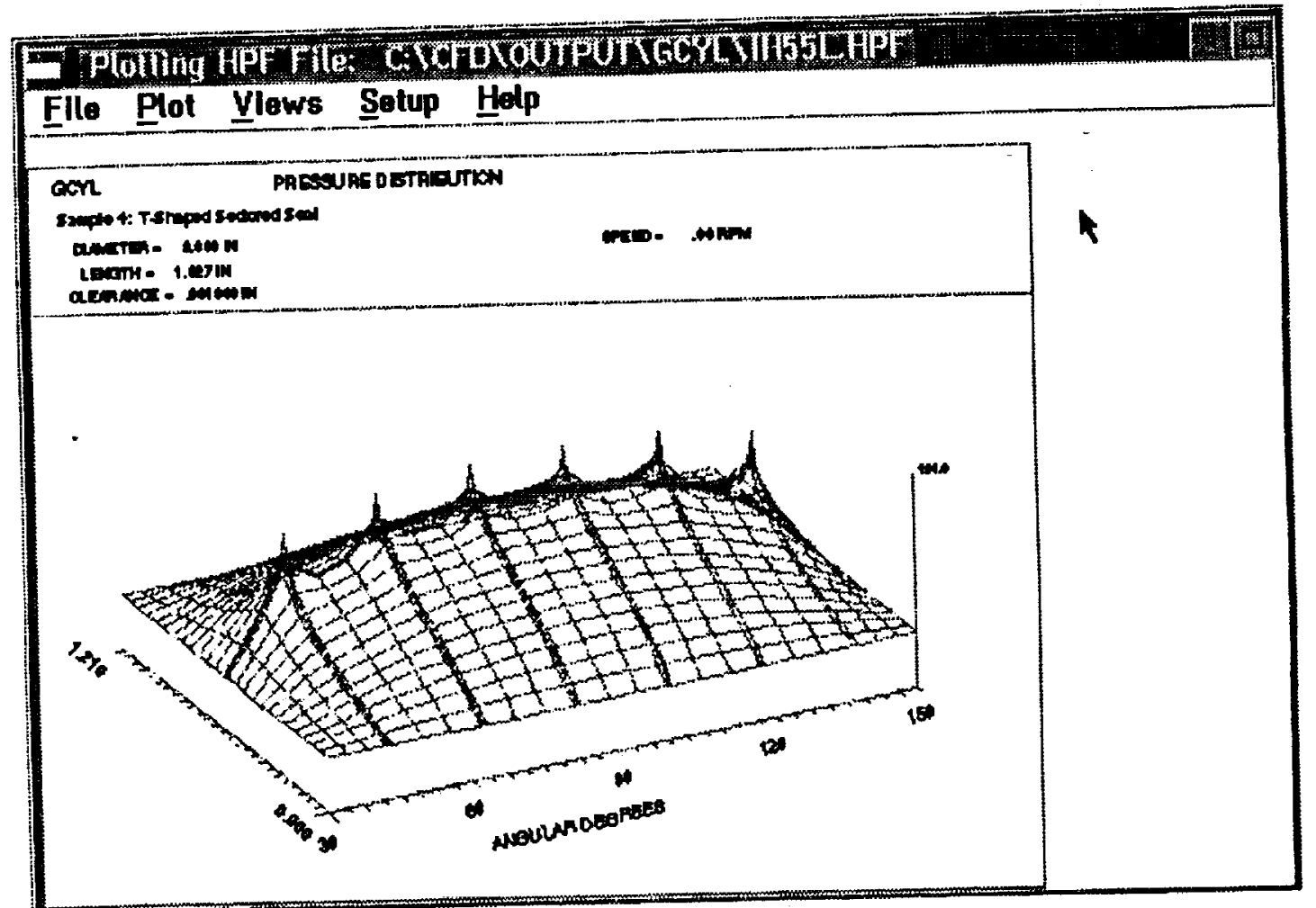

\section{Work In Progress - Future Plans}

New components to be added to the system:

- Labyrinth Seal Analysis (KTK) from the Air Force. Work about 70\% complete.

- Seal Dynamics Code (Face and Cylindrical Seals)

- Expert systems

- Enhancement of SPIRALI to include loss coefficients for spiral grooves

Operating System Considerations:

- Problems with conversion of user interface code to Unix

- Distributed Computing Environment (DCE) makes it possible to run programs on an OS/2 machine from a Unix workstation

- OS/2 to be available for Power PC RISC machines in April-May 1994.

- Ability to run both Unix and $O S / 2$ on the same machine at the same time on systems based on OSF Mach 3 kemel 